PROCEEDINGS OF THE

AMERICAN MATHEMATICAL SOCIETY

Volume 91, Number 1, May 1984

\title{
TWO PROBLEMS OF DOWKER
}

\author{
MARY ELLEN RUDIN
}

\begin{abstract}
An old question first raised by Hugh Dowker is asked again and two related problems are solved.
\end{abstract}

Question 1. Does there exist a set $X$ and a filter $F$ on $X$ having properties (1.a) and (1.b)?

(1.a) If $f: X \rightarrow F$, then there are $x \neq y$ in $X$ with $x \in f(y)$ and $y \in f(x)$.

(1.b) If $Y \subset X$, then there is $f: X \rightarrow F$ such that, if $y \in Y$ and $x \in X-Y$, either $x \notin f(y)$ or $y \notin f(x)$.

Question 2. Does there exist a set $X$ and, for each $x \in X$, a set $F_{x}$ of subsets of $X$ closed under finite intersections and having properties (2.a) and (2.b)?

(2.a) If $f(x) \in F_{x}$ for all $x \in X$, then there are $x \neq y$ in $X$ with $x \in f(y)$ and $y \in f(x)$.

(2.b) If $Y \subset X$, then there are $f(x) \in F_{x}$ for all $x \in X$, such that, for all $y \in Y$ and $x \in X-Y$, either $x \notin f(y)$ or $y \notin f(x)$.

Question 3. Does there exist a $T_{4}$, not paracompact, simplicial complex $K$, with the star of each vertex open? (We assume that the dimension of $K$ is 1 and that, if $x \neq y$ are vertices, there is at most one 1-simplex $[x, y]$ of $K$ having $x$ and $y$ as its end points. Observe that the vertices of $K$ testify that $K$ is not collectionwise Hausdorff; that is they cannot be covered by a family of disjoint open sets each containing only one vertex.)

A yes answer to Question 1 implies a yes answer to Question 2 which implies a yes answer to Question 3 (see Lemma 1). While trying to anwer the topological Question 3, Hugh Dowker was led to ask Question 1, the title question of [1].

So far as I know, Question 1 is still unanswered. The purpose of this note is to prove that the answer to Question 2 (and thus also Question 3) is yes. However, I conjecture that the answer to Question 1 is no.

LEMMA 1. If the answer to Question 2 is yes, so is the answer to Question 3.

Proof. Suppose $X$ and $\left\{F_{x} \mid x \in X\right\}$ give a yes answer to Question 2 .

We can assume that for each $x \in X, \bigcap F_{x}=\varnothing$. For otherwise, define $F_{x}^{\prime}=$ $\left\{f-\bigcap F_{x} \mid f \in F_{x}\right\}$. Clearly $\bigcap F_{x}^{\prime}=\varnothing$ and $\left\{F_{x}^{\prime} \mid x \in X\right\}$ has all the properties desired of $\left\{F_{x} \mid x \in X\right\}$. To see that (2.a) holds, assume on the contrary that for every $x \in X$, there is $f(x) \in F_{x}$ such that $x \neq y$ implies that either $y \notin\left(f(x)-\bigcap F_{x}\right)$ or $x \notin\left(f(y)-\bigcap F_{y}\right)$.

By (2.b) for all $x \in X$, there is $g_{x}(y) \in F_{y}$ for all $y \in X$ such that, if $y \neq x$ in $X$, then either $x \notin g_{x}(y)$ or $y \notin g_{x}(x)$. For each $x \in X$ suppose $f(x) \in F_{x}$; define $k(x)=f(x) \cap g_{x}(x)$. By (2.a) there are $x \neq y$ in $X$ such that $x \in k(y)$ and

Received by the editors June 17, 1983 and, in revised form, August 3, 1983.

1980 Mathematics Subject Classification. Primary 54D15, 54G20, 04A20; Secondary 03E35.

Key words and phrases. Normal, paracompact, filter, collectionwise Hausdorff. 
$y \in k(x)$. Since $y \in k(x) \subset g_{x}(x), x \notin g_{x}(y)$. But $x \in k(y) \subset f(y)$ so, since $g_{x}(y) \in F_{y}$, $x \in\left(f(y)-g_{x}(y)\right) \subset\left(f(y)-\bigcap F_{y}\right)$. Similarly $y \in\left(f(x)-\bigcap F_{x}\right)$. Thus (2.a) holds for $\left\{F_{x}^{\prime} \mid x \in X\right\}$, and we can assume that $\bigcap F_{x}=\varnothing$ for all $x \in X$.

If $x \neq y$ in $X$, choose a unit interval $[x, y]=[y, x]$ between $x$ and $y$ and let $d_{[x, y]}$ be the distance function on this interval. We let $K$ be the complex $X \cup\{[x, y] \mid x \neq$ $y$ in $X$; ; it remains to give the topology of $\bigcup K$ at the vertices. For $x \in X, A \in F_{x}$, and $0<\epsilon<\frac{1}{4}$, let

$$
\begin{aligned}
U_{A, \epsilon}(x)= & \{x\} \cup\left\{p \in[x, y] \mid x \neq y, y \in X-A, \text { and } d_{[x, y]}(x, p)<\epsilon\right\} \\
& \cup\left\{p \in[x, y] \mid x \neq y, y \in A, \text { and } d_{[x, y]}(x, p)<\left\{\frac{1}{2}\right\}+\epsilon\right\} .
\end{aligned}
$$

Let $\left\{U_{A, \epsilon}(x) \mid A \in F_{x}\right.$ and $\left.0<\epsilon<\frac{1}{4}\right\}$ be a basis for the topology of $K$ at $x$. This complex trivially has the desired properties.

THEOREM. The answer to Question 2 is yes.

Proof. Let $X$ be the set of all ordinals less than $c=|\mathbf{R}|$. Let $C=\{A \subset X \mid$ $A$ is countable $\}$ and $B=\{A \subset C \mid A$ is finite $\}$. Index $\left\{k: \mathbf{N}^{2} \rightarrow(X \times B)\right\}$ as $\left\{k_{x} \mid\right.$ $x<c\}$ in such a way that each $k$ is $k_{x}$ for $c$ many $x$ 's.

For all $y \in X$ we define

$$
A_{y}=\bigcup\left\{\bigcup A \mid A \text { is the second term of a member of the range of } k_{y}\right\} \text {. }
$$

If $x \in X$ and $Y \subset X$, we define $g_{x}(Y)=\{y \in X \mid x<y$ and there is $\langle x, A\rangle \in$ (range $\left.k_{y}\right)$ with $\left.\left(Y \cap A_{y}\right) \in \mathcal{A}\right\}$. Now define

$$
f_{x}(Y)=\left\{\begin{array}{l|l}
y \in X & \begin{array}{l}
\text { (a) } x \in Y \text { if and only if } y \in Y \text { or } \\
\text { (b) } x<y \text { and } y \in g_{x}(Y) \text { or } \\
\text { (c) } y<x \text { and } x \notin g_{y}(Y)
\end{array}
\end{array}\right\} .
$$

For $x \in X$, define $F_{x}=\left\{\bigcap_{Y \in Y} f_{x}(Y) \mid \mathcal{Y}\right.$ is a finite set of subsets of $\left.X\right\}$. Clearly $\left\{F_{x} \mid x \in X\right\}$ satisfies $(2 . \mathrm{b})$ : simply define $f(x)=f_{x}(Y)$. It remains to show that it satisfies (2.a).

Suppose that for every $x \in X, f_{x} \in F_{x}$. We find $x \neq y$ in $X$ with $x \in f_{y}$ and $y \in$ $f_{x}$. Let $\mathcal{Y}_{x}$ denote the finite set of subsets of $X$ such that $f_{x}=\bigcap\left\{f_{x}(Y) \mid Y \in \mathcal{Y}_{x}\right\}$.

We first choose a function $h: \mathbf{N}^{2} \rightarrow X$ by induction. For $i \in \mathbf{N}$ we let $H_{i}=$ $\left\{h\left(i^{\prime}, j\right) \mid i^{\prime}<i\right\}$ and we assume that $H_{i}$ is finite. Every $y \in X$ has an "ith characteristic function" $c_{y}:\left(\bigcup_{x \in H_{i}} y_{x}\right) \rightarrow 2$ defined by $c_{y}(Y)=1$ if and only if $y \in Y$. If $c_{1}, \ldots, c_{n}$ is a listing of the possible $i$ th characteristic functions for terms of $X-H_{i}$, for each $j \leq n$ choose $y \in X-H_{i}$ with $c_{y}=c_{j}$; then define $h(i, j)=y$. For $j>n$, define $h(i, j)=h(i, 1)$.

Let $H=$ range of $h$ and $y=\bigcup\left\{y_{x} \mid x \in H\right\}$. If $x \in H, x \in H_{i}$ for a minimal $i$; define $\mathcal{Y}_{x}^{*}=\mathcal{Y}_{x}-\bigcup\left\{\mathcal{Y}_{z} \mid z \in H_{i-1}\right\}$. For $Y \in \mathcal{Y}$ there is a unique $i=i_{Y} \in \mathbf{N}$ such that $Y \in \mathcal{Y}_{x}^{*}$ for some $x \in H_{i}$.

If $Y \neq Z$ in $y$ choose $a_{Y, Z}$ in either $Y-Z$ or $Z-Y$; then let $A=\left\{a_{Y, Z} \mid\right.$ $Y \neq Z$ in $\mathcal{Y}\}$. For $Y \in \mathcal{Y}$, let $A_{Y}=A \cap Y$. Observe that $A$ is countable, $A=$ $\bigcup\left\{A_{Y} \mid Y \in \mathcal{Y}\right\}$, and that if $Y \neq Z$ in $\mathcal{Y}$, then $A_{Y} \neq A_{Z}$. For each $x \in H$, let $\mathcal{A}_{x}=\left\{A_{Y} \mid Y \in \mathcal{Y}_{x}^{*}\right\}$, and define $k: \mathbf{N}^{2} \rightarrow(X \times B)$ by $k(i, j)=\left\langle x, A_{x}\right\rangle$ if $h(i, j)=x$.

Choose $y \in X$ such that $y>x$ for all $x \in H$ and $k_{y}=k$. Since $y_{y}$ is finite, so is $\mathcal{Y}^{*}=\left\{Y \in \mathcal{Y} \mid A_{Y}=(A \cap Z)\right.$ for some $\left.Z \in \mathcal{Y}_{y}\right\}$. Thus there is an $i \in \mathbf{N}$ such that $i>i_{Y}$ for all $Y \in \mathcal{Y}^{*}$. Since $y \in X-H$, there is $x \in H_{i+1}-H_{i}$ such that the $i$ th characteristic functions of $x$ and $y$ are the same. 
We show that $x \in f_{y}$ and $y \in f_{x}$, thus proving our theorem.

Recall that $f_{x}=\bigcap\left\{f_{x}(Y) \mid Y \in \mathcal{Y}_{x}\right\}$. If $Y \in\left(\mathcal{Y}_{x}-\mathcal{Y}_{x}^{*}\right)$, then $i_{Y} \leq i$ and, since $x$ and $y$ have the same $i$ th characteristic function, $x \in Y$ if and only $y \in Y$. Thus, by (a), $y \in f_{x}(Y)$. If $Y \in \mathcal{Y}_{x}^{*}$, then since $k_{y}=k,\left\langle x, A_{x}\right\rangle \in\left(\right.$ range $\left.k_{y}\right)$. Since $A_{Y}=A$, $A_{Y}=(Y \cap A) \in A_{x}$. By (b), since $x<y$ and $y \in g_{x}(Y), y \in f_{x}(Y)$. So $y \in f_{x}$.

To show that $x \in f_{y}$, suppose that $Y \in \mathcal{Y}_{y}$. By our choice of $i$, if $A \cap Y=A_{Z}$ for some $Z \in \mathcal{Y}$, then $i_{Z}<i$; so $A_{y} \cap Y=A \cap Y \notin \mathcal{A}_{x}$. Thus $y \notin g_{x}(Y)$ since $\left\langle x, \mathcal{A}_{x}\right\rangle$ is the only point in the range of $k$ having $x$ as its first coordinate. So, by (c), $x \in f_{y}(Y)$ and $x \in f_{y}$ and our proof is complete.

Lemma 2. The existence of a first countable, $T_{4}$, noncollectionwise Hausdorff space implies the answer to Question 2 is yes with the $F_{x}$ 's each being countable.

Proof. Let $S$ be the space and $X$ the closed discrete set of points in $S$ which cannot be separated; we assume without loss of generality, that the points of $X-S$ are isolated. For each $x \in X$, choose an open basis $U_{1}(x) \supset U_{2}(x) \supset \cdots$ for the topology at $x$ with $\overline{U_{1}(x)} \cap X=\{x\}$. For each $x \in X$, let $f_{n}(x)=\{y \in X-\{x\} \mid$ $\left.U_{n}(x) \cap U_{n}(y) \neq \varnothing\right\}$. Then, if $F_{x}=\left\{f_{n}(x) \mid n \in \mathbf{N}\right\},\left\{F_{x} \mid x \in X\right\}$ has the desired properties.

Comments on cardinalities. It has long been known that there is a model for ZFC in which there is a $T_{4}$, noncollectionwise Hausdorff space of countable character. In another model, all $T_{4}$, noncollectionwise Hausdorff spaces have character greater than $c$.

If $X$ and $\left\{F_{x} \mid x \in X\right\}$ yield a yes answer to Question 2, the manifold $K$ constructed as in the proof of Lemma 1 is a $T_{4}$, noncollectionwise Hausdorff space and each vertex $x$ has character $\leq\left|F_{x}\right|$ (or countable if $F_{x}$ is countable). By the proofs of Lemmas 1 and 2, the existence of $X$ and $\left\{F_{x} \mid x \in X\right\}$ yielding a yes answer to Question 2 with each $F_{x}$ countable is equivalent to the existence of a $K$ yielding a yes answer to Question 3 of countable character is equivalent to the existence of any $T_{4}$, noncollectionwise Hausdorff space of countable character; and all are undecidable in ZFC. Similarly the existence of $X$ and $\left\{F_{x} \mid x \in X\right\}$ yielding a yes answer to Question 2 with each $F_{x}$ of cardinality $\leq c$ or a $K$ yielding a yes answer to Question 3 of character $\leq c$ is undecidable in ZFC.

The $X$ of our Theorem has cardinality $c$. The cardinality of a particular $F_{x}$ depends on the choice of $\left\{k_{y} \mid y \in X\right\}$, but it could be as much as $2^{c}$. There is a consistency example with the cardinality of $X=\omega_{1}$ and all $F_{x}$ are countable. But for a real example, one cannot hope to do much better. However, none of these solutions of Question 2 are apt to lead to a solution of Question 1 since Dowker proves that any $X$ and $F$ yielding a yes answer to Question 1 must have the cardinality of $X$ greater than $\omega_{2}$. For completeness I give some of Dowker's results.

Comments on Question 1 (Dowker). Suppose that $X$ and $F$ yield a yes answer to Question 1 and that the cardinality $\alpha$ of $X$ is minimal for such to exist. Then $Y \in F$ implies $Y$ has cardinality $\alpha$ since $\{A \cap Y \mid A \in F\}$ would be an " $F$ " set for $Y$. Let $\beta$ be the minimal cardinality of a subset of $X$ whose complement is not in $F$. By (1.a), no nested sequence of members of $F$ has empty intersection. If $Y=\left\{y_{\gamma} \mid \gamma<\beta\right\} \subset X$ and for each $\delta<\beta, Y_{\delta}=X-\left\{y_{\gamma} \mid \gamma<\delta\right\}$, then $\left\{Y_{\delta} \mid \delta<\beta\right\}$ is a nested sequence of members of $F$; hence $\beta<\alpha$. Since there is such a $Y$ with $(X-Y) \notin F$, and $\beta<\alpha$ implies $Y \notin F, F$ is not an ultrafilter. Assuming $(X-Y) \notin$ 
$F$, let $f: X \rightarrow F$ satisfy (1.b) and, for $\delta<\beta$, define $Y_{\delta}^{*}=Y_{\delta} \cap f\left(Y_{\delta}\right)$. Then $Y_{\delta}^{*} \in F$ and $\bigcap_{\delta<\beta} Y_{\delta}^{*}=\varnothing$. It is impossible to have $\beta \leq \omega$ since then $Z_{\delta}=\bigcap_{\gamma \leq \delta} Y_{\gamma}^{*} \in F$ and $\left\{Z_{\delta} \mid \delta<\beta\right\}$ is a nested sequence with empty intersection. Hence $\omega<\beta<\alpha$ and $\alpha \geq \omega_{2}$.

\section{BIBLIOGRAPHY}

1. C. H. Dowker, A problem in set theory, J. London Math. Soc. 27 (1952), 371-374.

Department of Mathematics, University of Wisconsin, Madison, Wisconsin 53706 